\title{
Role of thrombolysis in haemodynamically stable patients with pulmonary embolism
}

\section{Alaistair Proudfoot, ${ }^{1}$ Dan Melley, ${ }^{2}$ Pallav L Shah ${ }^{1,2}$}

Pulmonary embolism (PE) is a common cardiopulmonary illness with an age and sex adjusted incidence of approximately 117 cases per 100000 person years. ${ }^{1}$ Incidence rises sharply after the age of 60 years in both men and women. While mortality rates exceed $15 \%$ in the 3 months following diagnosis, they are reduced to $2-10 \%$ if $\mathrm{PE}$ is diagnosed and treated promptly. ${ }^{2}{ }^{3}$ The majority of patients with PE are haemodynamically stable at presentation and receive anticoagulation with heparin. By contrast, thrombolysis (or embolectomy) is the accepted standard of care in those patients who present with haemodynamic compromise. ${ }^{4}$ Under such circumstances, mortality approaches $50 \%$ and although to date no study has been powered to show a mortality benefit, such treatment potentially outweighs the complication rate of $3 \% .^{25}$ Importantly, it is now recognised that there is another cohort of patients who, although haemodynamically stable, are nevertheless at an increased risk of death and who currently receive only standard anticoagulation regimens. To date, no consensus exists regarding the identification, appropriate risk stratification or indeed the further management of these patients. Those who favour thrombolysis for the treatment of PE argue that its use should be extended to the subgroup of patients with preserved systemic arterial pressure but evidence of right ventricular (RV) dysfunction. ${ }^{5}{ }^{6}$

The justification for considering thrombolysis in this group is based on the knowledge that mortality rates double in patients who have PE with preserved haemodynamic status but evidence of right heart dilatation. ${ }^{2}{ }^{7} \mathrm{RV}$ compromise is perceived to be a sign of possible impending haemodynamic instability and is a common cause of death. While

\footnotetext{
${ }^{1}$ Royal Brompton Hospital, London, UK; ${ }^{2}$ Chelsea and Westminster Hospital, London, UK

Correspondence to: Dr Pallav L Shah, Department of Respiratory Medicine, Royal Brompton Hospital, Sydney Street, London SW3 6NP, UK;

pallav.shah@imperial.ac.uk
}

case series suggest that RV dysfunction is present in up to $50 \%$ of patients with haemodynamically stable PE at presentation, ${ }^{2}{ }^{7}$ mortality data for such patients treated with heparin alone ranges from $0 \%$ to almost $30 \% .^{7-9}$ In contrast, it is clear from case series that patients with haemodynamic stability and a normal right heart on transthoracic echo have a mortality approaching $0 \%$ with appropriate anticoagulation. ${ }^{70}$ The challenge is therefore to identify the subgroup of patients with normotension who are likely to progress to RV failure and haemodynamic instability where the inherent risks of thrombolytic therapy may be justifiable.

Transthoracic echocardiographic assessment of RV dysfunction has traditionally been utilised as the firstline investigation for risk stratification of otherwise haemodynamically stable patients. ${ }^{7011}$ However, the assessment of RV dimension alone has an extremely poor specificity and positive predictive value. By contrast, a retrospective series of over 900 patients found that an RV/ left ventricular (LV) ratio greater than or equal to 0.9 was an independent predictive factor for hospital mortality (odds ratio (OR) 3.64). ${ }^{12}$ This measurement may increase the utility of echocardiography for identifying at risk patients. Nevertheless, the limited availability of emergency echocardiography in some centres, operator dependence, difficulty in visualising the right ventricle in patients with other respiratory pathology and finally poor specificity suggest that additional modalities are necessary for reliable risk stratification.

Computed tomography pulmonary angiography (CTPA) is already the investigation of choice for the diagnosis of suspected PE. More recently, given the limitations of echo, CTPA has also been proposed as a prognostic tool. One recent retrospective study found that analysis of $\mathrm{RV} / \mathrm{LV}$ ratio and azygous vein diameter (markers of clot load and RV dilatation) were the best CT predictors of survival, enabling correct prediction of survival in $89 \%$ of patients. ${ }^{13}$ This finding has been supported by other retrospective studies which suggest that alternative CTPA cardiac measurements may be used to reliably differentiate between survivors and non-survivors following PE. ${ }^{14} 15$

Serum levels of cardiac biomarkers, in particular troponin and brain natriuretic peptide (BNP), strongly correlate with RV dysfunction. They have a negative predictive value approaching 98\% for mortality and inhospital morbidity following $\mathrm{PE}$ and are therefore particularly useful for the identification of low risk patients. ${ }^{16}{ }^{17}$

A meta-analysis of 20 trials including 1985 patients assessed the predictive value of elevated troponin levels for early death and adverse outcome following PE. ${ }^{18}$ Overall, 122 of 618 patients with elevated troponin levels died (19.7\%; 95\% confidence interval (CI) 16.6 to 22.8) compared with 51 of 1367 with normal troponin levels (3.7\%; 95\% CI 2.7 to 4.7). Elevated troponin levels were significantly associated with short term mortality (OR 5.24) and death resulting from PE (OR 9.44). Furthermore, levels of troponin correlated with mortality in the subgroup of otherwise haemodynamically stable patients (OR 5.90). The results were consistent for both troponin I and $\mathrm{T}$.

Although BNP alone is a less sensitive independent predictor of death, algorithms using combinations of cardiac biomarkers in addition to echocardiographic evidence of RV dysfunction have been evaluated in the risk stratification of normotensive patients. One prospective study has shown that the combination of RV dysfunction with a BNP level $\geqslant 527 \mathrm{pg} / \mathrm{ml}$ showed prognostic value over RV dysfunction alone. Inhospital death rate and progression to shock were $55 \%$ and $31 \%$ in the two groups, respectively. ${ }^{19}$ A further study has demonstrated that normotensive patients with RV dysfunction on echo and elevated levels of both BNP and troponin have a 10fold higher risk of complications, including death. ${ }^{20}$ Finally, a study by Kostrubiec et al has demonstrated a mortality of $33 \%$ in patients with elevated levels of both NTproBNP and troponin T.

Clinical presentation with haemodynamic compromise is currently considered the most powerful predictor of death in $\mathrm{PE}$ and the associated mortality is justification for the use of thrombolytic therapy. There is, however, an emerging body of evidence to suggest that a subgroup of normotensive patients with RV dysfunction have mortality rates equivalent to patients presenting with haemodynamic compromise. The absolute cut-off levels for cardiac biomarkers, in particular BNP, require further 


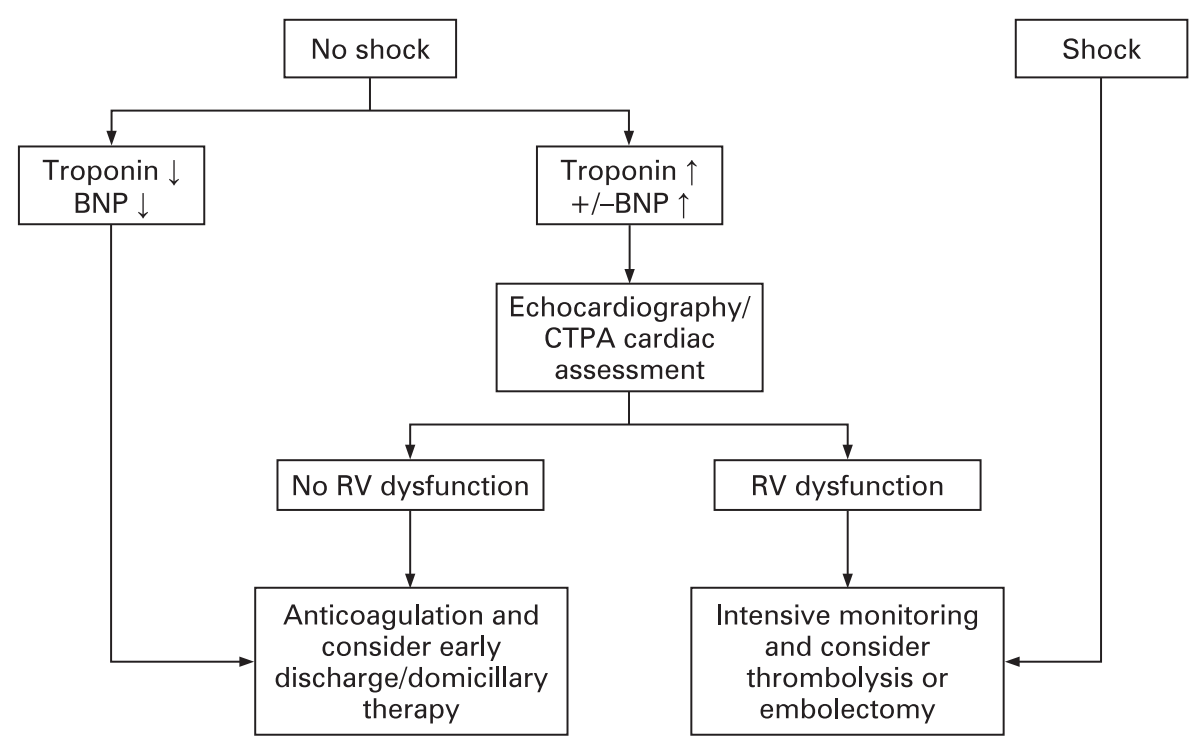

Figure 1 Treatment algorithm for pulmonary embolism. BNP, brain natriuretic peptide; CTPA, computed tomography pulmonary angiography; RV, right ventricular.

definition, and CTPA cardiac assessment needs to be prospectively validated. There is therefore a need for a large intention to treat trial in which CTPA and/or echo cardiac assessment are used together with the measurement of cardiac biomarkers to define the haemodynamically stable subgroup of patients who may benefit from more aggressive therapies (outlined in fig 1). This would facilitate the design of future international multicentre therapeutic trials.

In the interim, cardiac biomarkers together with physiological assessment (echo and CTPA) should be utilised first, to identify patients suitable for a brief hospital stay or even outpatient management, and second, to identify the potentially high risk group of normotensive patients who may merit intensive monitoring for the initial phase of their illness.

Competing interests: None.

Thorax 2008;63:853-854. doi:10.1136/thx.2008.103077

\section{REFERENCES}

1. Heit JA. The epidemiology of venous thromboembolism in the community: implications for prevention and management. J Thromb Thrombolysis 2006;21:23-9.

2. Goldhaber SZ, Visani L, De Rosa M. Acute pulmonary embolism: clinical outcomes in the International Cooperative Pulmonary Embolism Registry (ICOPER). Lancet 1999;353:1386-9.

3. Carson JL, Kelley MA, Duff A, et al. The clinical course of pulmonary embolism. $N$ Engl J Med 1992:326:1240-5.

4. Buller HR, Agnelli G, Hull RD, et al. Antithrombotic therapy for venous thromboembolic disease: the Seventh ACCP Conference on Antithrombotic and Thrombolytic Therapy. Chest 2004;126:401S-28.

5. Konstantinides S, Geibel A, Olschewski M, et al. Association between thrombolytic treatment and the prognosis of hemodynamically stable patients with major pulmonary embolism: results of a multicenter registry. Circulation 1997;96:882-8.

6. Konstantinides S. Thrombolysis in submassive pulmonary embolism? Yes. J Thromb Haemost 2003;1:1127-9.

7. Grifoni S, Olivotto I, Cecchini P, et al. Short-term clinical outcome of patients with acute pulmonary embolism, normal blood pressure, and echocardiographic right ventricular dysfunction. Circulation 2000;101:2817-22.
8. Kostrubiec M, Pruszczyk P, Bochowicz A, et al. Biomarker-based risk assessment model in acute pulmonary embolism. Eur Heart $J$ 2005;26:2166-72.

9. Hamel E, Pacouret G, Vincentelli D, et al. Thrombolysis or heparin therapy in massive pulmonary embolism with right ventricular dilation results from a 128-patient monocenter registry. Chest 2001:120:120-5.

10. Kasper W, Kostantinides S, Geibel A, et al. Prognostic significance of right ventricular afterload stress detected by echocardiography in patients with clinically suspected pulmonary embolism. Heart 1997; 77:346-9.

11. Kucher N, Rossi E, De Rosa M, et al. Prognostic role of echocardiography among patients with acute pulmonary embolism and a systolic arterial pressure of $90 \mathrm{~mm} \mathrm{Hg}$ or higher. Arch Intern Med 2005; 165:1777-81.

12. Frémont B, Pacouret G, Jacobi D, et al. Prognostic value of the echocardiographic right/left ventricular end-diastolic diameter ratio in patients with acute pulmonary embolism: Results from a monocenter registry of 1,416 patients. Chest 2008;133:358-62.

13. Ghaye B, Ghuysen A, Willems V, et al. Severe pulmonary embolism:pulmonary artery clot load scores and cardiovascular parameters as predictors of mortality. Radiology 2006;239:884-91.

14. van der Meer RW, Pattynama PM, van Strijen MJ, et al. Right ventricular dysfunction and pulmonary obstruction index at helical CT: prediction of clinical outcome during 3-month follow-up in patients with acute pulmonary embolism. Radiology 2005;235:798-803.

15. Schoepf UJ, Kucher N, Kipfmueller F, et al. Right ventricular enlargement on chest computed tomography: a predictor of early death in acute pulmonary embolism. Circulation 2004;110:3276-80.

16. Jiménez Castro D, Díaz G, Molina J, et al. Troponin I and risk stratification of patients with acute non massive pulmonary embolism. Eur Respir J 2007 (Epub ahead of print 19 Dec 2007).

17. Kucher N, Printzen G, Doernhoefer T, et al. Low probrain natriuretic peptide levels predict benign clinical outcome in acute pulmonary embolism. Circulation 2003;107:1576-8.

18. Becattini C, Vedovati MC, Agnelli G. Prognostic value of troponins in acute pulmonary embolism: a metaanalysis. Circulation 2007;116:427-33.

19. Pieralli F, Olivotto I, Vanni S, et al. Usefulness of bedside testing for brain natriuretic peptide to identify right ventricular dysfunction and outcome in normotensive patients with acute pulmonary embolism. Am J Cardiol 2006;97:1386-90.

20. Binder L, Pieske B, Olschewski M, et al. N-terminal pro-brain natriuretic peptide or troponin testing followed by echocardiography for risk stratification of acute pulmonary embolism. Circulation 2005;112:1573-9. 